\title{
MATHEMATICS OF COMPUTATION
}

Beginning with the January 1960 issue, the title of Mathematical Tables and other Aids to Computation has been changed to Mathematics of Computation. The new name was unanimously adopted by the Editorial Committee in order to reflect the broadened scope of the Journal, which has expanded to meet the need in this country for a publication devoted to numerical analysis and computation. As stated in the announcement of the October issue, the new title in no way represents a diminished interest in mathematical tables, which will continue to be a subject of major emphasis, as in the past. It recognizes, however, an increased interest in other areas in the field of Mathematics of Computation, which have grown in importance and in which rapid advances are being made in the present era of technological progress.

The first issue of Mathematical Tables and other Aids to Computation appeared in January 1943, more than one year prior to the completion of the first large scale computer - the Harvard Automatic Sequence Controlled Calculator. It is quite evident, however, that Professor R. C. Archibald, when he founded the journal, had to a large extent foreseen the great progress which was to follow in the development of instruments for computation. In the introductory remarks which he published in the first issue he established as one of the principal goals of the Journal, "to serve as a clearing-house for information" concerning tools of computation which have been "vastly multiplied during the past decade." He further speaks of these tools, or accounts of them, which "are to be found in an enormous international range of book, pamphlet, and periodical publication, not only in the fields of Pure Mathematics, Physics, Statistics, Astronomy, and Navigation, but also in such fields as Chemistry, Engineering, Geodesy, Geology, Physiology, Economics, and Psychology."

Indeed during the lifetime of this journal we have witnessed the development of one of the most promising technological tools ever devised by man-the electronic digital calculator. During this period the speed of digital calculators has increased one-hundred-thousand-fold. High-speed calculators are being used to translate from one language to another, to track satellites, to compose "symphonies," to design nuclear reactors, to forecast weather, to monitor this country's early warning system, to play chess, to simulate the motion of a submarine, or to compute fall-out patterns. This is but a small sample of the many complex tasks which are being performed by these devices. However, we are only in the initial stages of this development. It is not yet sixteen short years since the first largescale automatic calculator has been placed in operation. Mathematics of Computation will in part be devoted to the exploration of new areas of applications for highspeed computer devices in every field of human endeavor.

Substantial progress has also been made in the development of numerical methods. For instance, until very recently it has not been feasible to obtain numerical solutions for most types of systems of ordinary and partial differential equations. This is no longer the case. Numerical solutions of complex systems of differential and integral equations-based on the methods of finite differences and aided by the availability of high-speed calculators-are now readily attainable. 
This constitutes a major advance in applied mathematics. The mathematical equations which govern the solution of problems in many areas of engineering and theoretical physics are systems of differential equations. Supersonic and subsonic aerodynamics, nuclear reactor design theory, heat transfer, propagation of electromagnetic and acoustic waves are but a few illustrative fields which fall in this category. Mathematics of Computation will be devoted to advances in numerical analysis with the view toward further expansion of our capabilities to obtain numerical solutions to systems of mathematical relations of any type.

Many new mathematical disciplines are in the process of development which are required to advance the theory and foster the application of numerical methods. Information theory, the logic of automata, operational analysis, the theory of games, and linear programming are a few examples of such fields of current interest. These and many other mathematical developments will be required to comprehend clearly and utilize fully the vast potential of modern computational devices. Mathematics of Computation will be available for the publication of advances in modern mathematical theories related to computation.

In view of the phenomenally rapid progress in the development of computational devices and the equally impressive advances in numerical analysis, we look forward with keen interest and considerable expectation toward the future, to witness the exciting technological progress that will result from this major advance or break-through in applied mathematics. With this entire field of modern mathematics, embracing

(1) advances in numerical analysis,

(2) the application of numerical methods and high-speed calculator devices,

(3) the computation of mathematical tables,

(4) the development of new mathematical disciplines related to computation,

(5) the theory of high-speed calculating devices and other aids to computation, we have associated the title,

Mathematics of Computation.

H. P. 